\title{
Biosynthesis of the Tricyclic Aromatic Type II Polyketide Rishirilide: New Potential Third Ring Oxygenation after Three Cyclization Steps
}

\author{
Ahmad Alali ${ }^{1}$. Lin Zhang ${ }^{2}$. Jianyu $\mathrm{Li}^{3} \cdot$ Chijian Zuo $^{1} \cdot$ Dimah Wassouf $^{1} \cdot$ Xiaohui Yan $^{1}$. Philipp Schwarzer ${ }^{1}$. \\ Stefan Günther ${ }^{3}$. Oliver Einsle ${ }^{2} \cdot$ Andreas Bechthold $^{1}$ (1)
}

Received: 17 November 2020 / Accepted: 5 March 2021 / Published online: 24 March 2021

(c) The Author(s) 2021

\begin{abstract}
Rishirilides are a group of PKS II secondary metabolites produced by Streptomyces bottropensis Gö C4/4. Biosynthetic studies in the past have elucidated early and late steps of rishirilide biosynthesis. This work is aiming to solve the remaining steps in the rishirilide biosynthesis. Inactivation of the cyclase gene rslC3 in Streptomyces bottropensis resulted in an interruption of rishirilide production. Instead, accumulation of the tricyclic aromatic galvaquinones was observed. Similar results were observed after deletion of $r s \mathrm{O} 4$. Closer inspection into RslO4 crystal structure in addition to site-directed mutagenesis and molecular dynamic simulations revealed that RslO4 might be responsible for quinone formation on the third ring. The RslO1 three-dimensional structure shows a high similarity to FMN-dependent luciferase-like monooxygenases such as the epoxy-forming MsnO8 which acts with the flavin reductase MsnO3 in mensacarcin biosynthesis in the same strain. The high sequence similarity between $\mathrm{RslO} 2$ and $\mathrm{MsnO} 3$ suggests that $\mathrm{RslO} 2$ provides RslO1 with reduced FMN to form an epoxide that serves as substrate for RslO5.
\end{abstract}

Keywords Rishirilide $\cdot$ Cyclization $\cdot$ Oxygenation $\cdot$ Epoxidation $\cdot$ CRISPR-Cas9

$\begin{array}{ll}\begin{array}{l}\text { Abbreviations } \\ \text { Rsl gene cluster }\end{array} & \begin{array}{l}\text { Rishirilide gene cluster } \\ \text { Polyketide Synthase type II } \\ \text { PKS II }\end{array} \\ \text { CRISPR-Cas9 } & \begin{array}{l}\text { Clustered Regularly Interspaced Short } \\ \text { Palindromic Repeats system } \\ \text { Flavinmononucleotide }\end{array} \\ \text { HPLC/ESI-MS } & \begin{array}{l}\text { High-Performance Liquid Chromatog- } \\ \text { raphy/Electrospray Ionization }\end{array} \\ \text { IMAC Ni-NTA } & \begin{array}{l}\text { Immobilized Metal Affinity Chromatog- } \\ \text { raphy Nickle-Nitrilotriacetic Acid }\end{array} \\ \text { PDB } & \begin{array}{l}\text { Protein Data Bank } \\ \text { Area Under the Curve }\end{array} \\ \text { AUC } & \text { Cyclase/Aromatase } \\ \text { CYC/ARO } & \end{array}$

Andreas Bechthold

andreas.bechthold@pharmazie.uni-freiburg.de

1 Institute of Pharmaceutical Biology and Biotechnology, Albert-Ludwigs-Universität, Stefan-Meier-Straße 19, 79104 Freiburg, Germany

2 Institute of Biochemistry, Albert-Ludwigs-Universität, Albertstr 21, 79104 Freiburg, Germany

3 Institute of Pharmaceutical Bioinformatics, Albert-Ludw igs-Universität, Hermann-Herder-Str 9, 79104 Freiburg, Germany
SDS-PAGE Sodium Dodecyl Sulphate-Polyacryla-

His-Tag Histidine tag

NAD Nicotinamide Adenine Dinucleotide

Apramycin $^{\mathrm{R}}$

Spectinomycin $^{R} \quad$ Spectinomycin Resistance

SDM

IPTG Isopropyl $\beta$-D-1-thiogalactopyranoside

\section{Introduction}

Rishirilide is a PKS-II secondary metabolite isolated from two soil inhabitant Streptomyces species [S. rishiriensis OFR-1056 and S. bottropensis Gö C4/4 (S. bottropensis)] and one deep-sea species (S. olivaceus SCSIO T05) [1,2]. This compound has been shown to have moderate biological activities including the inhibition of $\alpha 2$-macroglobulin, glutathione S-transferase, and asparagine-tRNA synthase, whose roles in cancer and thrombosis have been sufficiently characterized over the past years [3].

Structurally, rishirilide is a tricyclic partially aromatic polyketide with an unusual isopentyl sidechain and a carbonyl group on the third ring. This makes it an intriguing 
target of the biosynthetic pathway studies. Schwarzer et al. identified the origins of the tricyclic backbone and some other substitutions in rishirilide B. The analysis of labelled compound using Nuclear Magnetic Resonance (NMR) spectroscopic revealed that the origin of isopentyl sidechain is isobutyryl-CoA, which is derived from valine. The tricyclic skeleton is synthesized from nine acetate units, and the methyl group is derived from a decarboxylation of one acetate unit [4].

In the course of our rishirilide biosynthetic pathway studies, a remarkable amount of information on early and tailoring steps has been recently elucidated and published. The polyketide synthase RslK4 and the putative 3-oxoacyl-ACP reductase $\mathrm{RslO} 3$ are involved in the generation of the unusual starter unit, and the early acting ketoreductase RslO10 is involved in first ring cyclization/aromatization [5].

Gene inactivation studies and biological assays yielded a detailed insight into the rearrangement of the oxidative carbon backbone in rishirilide biosynthesis. The flavindependent RslO5 is responsible for epoxide-opening in its anthraquinonic substrate. Subsequently, flavin monooxygenase RslO9 mediates the lactone-forming Baeyer-Villiger oxidation, and the resulted rearranged intermediate is converted to rishirilide A via the ketoreductase RslO8. Rishirilide $\mathrm{A}$ is then converted to rishirilide $\mathrm{B}$ via $\mathrm{Rs} 1 \mathrm{O} 9$ and water release [6].

S. albus containing a cosmid with genes of the rishirilide biosynthetic gene cluster $(\cos 4)$ was producing rishirilide B as major compound. $S$. albus containing cos4 with a deletion in $r s l O 4$ was producing galvaquinone A and B. And $S$. albus containing $\cos 4$ with a deletion in $r s l O l$ was producing galvaquinone $\mathrm{A}$ as major compound. We have proposed that galvaquinone $\mathrm{A}$ and $\mathrm{B}$ are shunt products resulting from unstable intermediates [5].

In this study the involvement of one cyclase and two oxygenase in rishirilide biosynthesis was investigated. For this purpose, excision of $r s l C 3$ gene using the markerless timesaving CRISPR-Cas9 system, inactivation of $r$ slO4 gene using Redirect ${ }^{\circledR}$ technology, and crystallization of RslO4 and RslO1 proteins were carried out. Molecular dynamics simulations and site-directed mutagenesis were used to predict the roles of $\mathrm{RslO} 4$ and $\mathrm{RslO} 1$ in quinone formation and epoxidation on the third ring, respectively.

\section{Materials and Methods}

\section{Bacterial Strains}

S. bottropensis and Streptomyces albus J1074, E. coli DH5 $\alpha$ for plasmid propagation, and E. coli ET12567/pUZ8002 for intergeneric conjugation were used. Long-term storage of
Streptomyces and E. coli cells was done in glycerol $15 \%$ $(V / V)$ at $-20^{\circ} \mathrm{C}$ and $-80^{\circ} \mathrm{C}$, respectively.

\section{Bacterial Cultivation and Expression Media}

TSB medium (Caso Boullion $30 \mathrm{~g} / \mathrm{l}$ ) for Streptomyces cultivation. LB-medium (Luria Bertani $20 \mathrm{~g} / \mathrm{l}$ ) and LB-agar medium (LB medium with agar-agar $21 \mathrm{~g} / \mathrm{l}$ ) for $E$. coli strains cultivation. $\mathrm{SG}^{+}$medium (glucose $20 \mathrm{~g} / \mathrm{l}$, soytone $10 \mathrm{~g} / \mathrm{l}, \mathrm{CaCO}_{3} 2 \mathrm{~g} / \mathrm{l}, \mathrm{CoCl}_{2} 0.1 \% \mathrm{~m} / \mathrm{V}$, L-valin $2.34 \mathrm{~g} / \mathrm{l}$ ) for secondary metabolites production in S. albus strains. YmPG medium (yeast extract $4 \mathrm{~g} / \mathrm{l}$, pepton from casein $1 \mathrm{~g} / \mathrm{l}$, glucose $10 \mathrm{~g} / \mathrm{l}$, malt extract $10 \mathrm{~g} / \mathrm{l}, \mathrm{MgCl}_{2} \cdot 6 \mathrm{H}_{2} \mathrm{O} 2 \mathrm{~g} / \mathrm{l}$, L-valin $5 \% 40 \mathrm{ml} / \mathrm{l}$ ) for secondary metabolites production in $\mathrm{S}$. bottropensis strains. MS-agar medium (mannitol $20 \mathrm{~g} / \mathrm{l}$, soya flour $20 \mathrm{~g} / \mathrm{l}$, agar-agar $21 \mathrm{~g} / \mathrm{l}, \mathrm{MgCl}_{2} 10 \mathrm{mM}$ ) for intergeneric conjugation. $2 \times \mathrm{YT}$ medium (tryptone $16 \mathrm{~g} / \mathrm{l}, \mathrm{NaCl}$ $5 \mathrm{~g} / \mathrm{l}$, yeast extract $10 \mathrm{~g} / \mathrm{l}$ ) for heat shock of $S$. bottropensis spores preparing for intergeneric conjugation. All media were freshly prepared, initial $\mathrm{pH}$ values were adjusted to 7.2 and directly autoclaved (Tuttnauer Systec 5075 ELV, Germany).

\section{CRISPR-Cas9 System for Gene Deletion of rs/C3 in S. bottropensis}

The system used in this study has been developed and optimized for actinomycetes in The Novo Nordisk Foundation Center for Biosustainability [7]. Two cloning procedures were required to prepare each construct: cloning of singleguide RNA (sgRNA) scaffold specific for the target gene between NcoI and SnaBI restriction sites and cloning of Homologous-Directed Repair Template (HDR Template) into StuI restriction site. sgRNA scaffold was designed by fusing CRISPR RNA (crRNA, 20 nt specific to a unique sequence in the target gene) with the associated sgRNA core sequence (trans-activating CRISPR RNA, tracr RNA). CRISPOR tool offered online from Zhang Lab was used for detecting and evaluating the $20 \mathrm{nt}$ crRNA guide sequences ending with-NGG as Protospacer Adjacent Motifs (PAM) for targeting $r s l C 3$. In order to prepare the HDR-Template, 800 bps upstream - and 800 bps downstream the target gene was PCR-amplified with 40 complementary base pairs from the sides to be connected, then the two PCR products were joined by overlapping PCR technique using only the two primers annealed from the other sides (Table S1).

To transform the constructs into $S$. bottropensis, E. coli ET12567/pUZ8002 was grown to an $\mathrm{OD}_{600}=0.4-0.6$ in the presence of apramycin $(50 \mu \mathrm{g} / \mathrm{mL})$ and kanamycin $(50 \mu \mathrm{g} /$ $\mathrm{mL})$. After centrifugation cells were washed and mixed with $50{ }^{\circ} \mathrm{C}$ heat-shocked spores on MS-agar plates supported with $\mathrm{MgCl}_{2}(10 \mathrm{mM})$ at $28^{\circ} \mathrm{C}$ for $16-20 \mathrm{~h}$. After that, the plates were overlaid with apramycin $(50 \mu \mathrm{g} / \mathrm{mL})$ and fosfomycin 
$(200 \mu \mathrm{g} / \mathrm{mL})$ and incubated again at $28^{\circ} \mathrm{C}$ until the exconjugants appeared. During replica-plating of the exconjugants, endonuclease activity of Cas9 was induced by thiostrepton $(1 \mu \mathrm{g} / \mathrm{mL})$. Finally, deletion of the target gene in the genomic DNA of some exconjugants was verified by PCR and sequencing.

\section{Complementation of $\Delta r s / C 3$ Mutant}

After deletion of rslC3 gene in S. bottropensis, the temperature sensitive plasmid was eliminated by growing at $37^{\circ} \mathrm{C}$ in liquid TSB media till the cells lost the resistance to apramycin. Following this, S. bottropensis- $\Delta r s l C 3$ spores were transformed with the integrative expression vector pSET152 harboring $r s l C 3$ gene (cloned between XbaI and EcoRV restriction sites) by intergeneric conjugation taking into consideration that pSET152 vector carries apramycin resistance marker (Table $\mathrm{S} 1$ ).

\section{Redirect $^{\oplus}$ Technology for $r s / 04$ Gene Editing in S. bottropensis}

The protocol used for this study has been developed by Gust B, Kieser T, and Chater KF [8]. The procedure was adapted to this work with some modifications. For this purpose, Cosmid 4 prepared in our laboratory by Xiaohui Yan from pOJ436 backbone with the whole gene cluster of rishirilide (Rsl gene cluster) was used after replacing the integrase gene with ampicillin ${ }^{\mathrm{R}}$ marker by Olga Tsypik and replacing apramycin ${ }^{\mathrm{R}}$ marker with spectinomycin ${ }^{\mathrm{R}}$ marker in this work. The gene $r s l O 4$ was replaced by apramycin cassette using homologous recombination in an E. coli strain containing $\mathrm{pBAD} \alpha \beta \gamma$ plasmid in the presence of L-arabinose $(0.01 \mathrm{M})$. pLEERE and pCDF Deut-1 vectors were used as PCR templates for the amplification of apramycin ${ }^{\mathrm{R}}$ and spectinomycin $^{\mathrm{R}}$ cassettes, respectively. Forward and reverse primers were designed as mentioned in the protocol from Gust and his colleagues with 60 nt (40 nt complementary to the flanking regions of the target gene and $20 \mathrm{nt}$ corresponding to the cassette sequence). PCR amplification method mentioned in the protocol was followed as well. After replacing $r$ slO4 gene with apramycin ${ }^{\mathrm{R}}$ cassette, the cosmid was transformed into $S$. bottropensis by intergeneric conjugation using E. coli ET12567/pUZ8002 strain. The overnight growing $E$. coli cells containing the prepared nonintegrative $r$ slO4-mutant $\mathrm{Cos} 4$ in the presence of apramycin $(50 \mu \mathrm{g} / \mathrm{mL})$, spectinomycin $(100 \mu \mathrm{g} / \mathrm{mL})$, and kanamycin $(50 \mu \mathrm{g} / \mathrm{mL})$ were mixed with the mycelia on MS-agar supported with $\mathrm{MgCl}_{2}(10 \mathrm{mM})$ at $28{ }^{\circ} \mathrm{C}$ for $8 \mathrm{~h}$. Then, the plates were overlaid with apramycin $(50 \mu \mathrm{g} / \mathrm{mL})$ and fosfomycin $(200 \mu \mathrm{g} / \mathrm{mL})$ but not with spectinomycin because the cosmid was suicidal after undergoing double cross-over with $S$. bottropensis genomic DNA. Replacement of $r s l O 4$ in the genomic DNA of $S$. bottropensis with apramycin cassette was verified by PCR depending on the difference in length between rslO4 (300 bps) and apramycin cassette (1045 bps) (Table S1).

\section{Induction of Secondary Metabolites Production in S. bottropensis $\Delta r s / 04$ by Positive Regulators}

We recently described that $S$. albus $\mathrm{J} 1074$ containing cos 4 and a plasmid containing all three regulatory genes $r s l R 1$, $r s l R 2$ and $r s l R 3$ produced more rishirilide B than $S$. albus J1074 containing only $\cos 4$ [5]. In an attempt to find the natural substrate of RslO4 enzyme, the $\Delta r s l O 4$ mutant of $S$. bottropensis was transformed with the replicative vector pUWL-H harboring the three regulatory genes $r s l R 1$, $r s l R 2$ and $r s l R 3$. Intergeneric conjugation and overlay with apramycin $(50 \mu \mathrm{g} / \mathrm{mL})$, hygromycin B $(50 \mu \mathrm{g} / \mathrm{mL})$, and fosfomycin $(200 \mu \mathrm{g} / \mathrm{mL})$ were carried out.

\section{Expression and Extraction of Secondary Metabolites of Streptomyces}

YmPG and $\mathrm{SG}^{+}$media were used in this study for the fermentation and secondary metabolite production in S. bottropensis and $S$. albus strains, respectively. Prior to fermentation, precultures of the strains were prepared in TSB medium in the presence of appropriate antibiotics at $28^{\circ} \mathrm{C} / 180 \mathrm{rpm}$ in the shaking incubator (Infors HT, Switzerland) for 2-3 days till the formation of well-growing cultures. The fermentation medium was inoculated with $1 \%$ of the preculture and then incubated at $28{ }^{\circ} \mathrm{C} / 180 \mathrm{rpm}$ for 5 days. Extraction of the secondary metabolites was carried out by ethyl acetate at $\mathrm{pH} 4$, subsequently ethyl acetate was evaporated, and the crude extracts were solved in methanol (100\%) and analyzed by HPLC/ESI-MS (Table S2).

\section{Overexpression and Purification of RsIO1 and RsI04 Proteins}

Each of $\mathrm{rslO}_{4}$ and $\mathrm{rslO} 1$ genes were amplified using cosmid 4 as a template. The amplified PCR products were cloned into the expression vector pET21a(+) (Novagen, Merck, Darmstadt, Germany) between NdeI and XhoI restriction sites to produce $\mathrm{C}$-terminal His-tag recombinant proteins (Table S1). The resulted constructs were verified by PCR and DNA sequencing. Expression was performed in $E$. coli BL21 (DE3) Star cells (Invitrogen, Thermo Fisher, Waltham, USA). The precultures of E. coli BL21 (DE3) cells containing the constructs were prepared in LB-media at $37{ }^{\circ} \mathrm{C} / 180 \mathrm{rpm}$ in the presence of $100 \mu \mathrm{g} / \mathrm{ml}$ ampicillin overnight. Thereafter, the growing cells were inoculated in $3 \mathrm{~L} \mathrm{LB}$-media for each protein and incubated at the same conditions. By reaching an $\mathrm{OD}_{600}$ of 0.6 , the 
overexpression was induced by addition of isopropyl $\beta$-D1-thiogalactopyranoside (IPTG, Carl Roth, Karlsruhe, Germany) in a concentration of $0.1 \mathrm{mM}$ and the media were incubated again at $20^{\circ} \mathrm{C} / 180 \mathrm{rpm}$ for $12 \mathrm{~h}$. Cells were harvested $12 \mathrm{~h}$ after induction by Centrifugation at $5000 \times g$ for 10 min (Rotina 380P, Hettich, Germany). The cell pellet was re-suspended in four pellet volumes of lysis buffer [Tris- $\mathrm{HCl}(\mathrm{pH} 8.0,50 \mathrm{~mm}), \mathrm{NaCl}(300 \mathrm{~mm})]$. The cells were lysed using French Pressure cell $(40 \mathrm{~K})$ at a pressure of 800 to 1000 psi. The suspension was mixed with $10 \mu \mathrm{L}$ of DNase and incubated for $15 \mathrm{~min}$ on ice. Thereafter, the protein containing soluble fraction was separated by centrifugation at $10,000 \times g, 4{ }^{\circ} \mathrm{C}, 1 \mathrm{~h}$ (Avanti J-6000 Rotor JA-10, Bechmann Coulther, Germany). This fraction was further purified by IMAC Ni-NTA affinity chromatography as follows: The protein sample was loaded on a $5 \mathrm{~mL}$ HisTrap $^{\text {TM }}$ FF column (GE Healthcare GmbH, Solingen, Germany), which was previously equilibrated with lysis buffer, using a flow rate of $0.5 \mathrm{ml} / \mathrm{min}$. The column was then stepwise washed with 5-15\% elution buffer [Tris-HCl (pH 8.0, $50 \mathrm{~mm}), \mathrm{NaCl}(300 \mathrm{~mm})$, imidazole $(500 \mathrm{~mm})]$ at a flow rate of $2 \mathrm{ml} / \mathrm{min}$. The desired protein was eluted with $50 \%$ elution buffer, analyzed by SDS-PAGE and its stability was observed. Fractions containing the desired protein were pooled and concentrated to a final volume of $1 \mathrm{~mL}$ by centrifugation in a Vivaspin concentrator (Sartorius, Göttingen, Germany). Finally, the protein was purified with a size exclusion HiLoad ${ }^{\circledR}$ 16/60 Superdex ${ }^{\circledR} 200$ column (GE Healthcare, Solingen, Germany), pre-equilibrated with buffer [Tris- $\mathrm{HCl}$ (pH 7.5, $20 \mathrm{~mm}), \mathrm{NaCl}(150 \mathrm{~mm})]$.

\section{Crystallization and Structure Determination of RsI01}

After size exclusion chromatography (Superdex S200 16/60) in buffer (20 mM Tris pH 8.0, $150 \mathrm{mM} \mathrm{NaCl}$ ), RslO1 protein was concentrated to $24 \mathrm{mg} / \mathrm{ml}$ before setting up initial screens using sitting drop vapor-diffusion method at $20^{\circ} \mathrm{C}$. A drop of $0.6 \mu \mathrm{l}$ in total with different protein to reservoir ratios $(25 \%, 50 \%)$ was set and equilibrated again reservoir solutions $(50 \mu \mathrm{l})$, using an automatic crystallization dropset system (Oryx Nano, Douglas Instrument). Large crystal clusters were grown for four weeks in $0.2 \mathrm{M}$ calcium chloride, 0.1 M HEPES pH 7.0 and 33\% polyethylene glycol conditions. Then these crystals were crashed for seeding experiment by using Oryx Nano. Then nice single crystals were grown in conditions with $0.1 \mathrm{M}$ HEPES pH 7.5 and $36 \%$ polyethylene glycol 600 after one week and kept growing for 4 weeks before harvesting. Crystals were mounted on cryo-loops and flash frozen in liquid nitrogen prior to data collection. Datasets to $1.6 \AA$ were collected at beamline X06SA (PXI) at Swiss Light Source (Villigen, Switzerland) with the PILATUS $6 \mathrm{M}$ detector. The data were processed using iMosflm [9] and XDS package [10], the crystal was assigned to the space group $\mathrm{P} 2{ }_{1} 2_{1} 2$ with unit cell dimensions $a=50.89, b=81.77 \AA$ and $C=160.61 \AA$.

RslO1 monooxygenase crystal structure was determined by automated molecular replacement in MrBUMP [11] using monomer of a monooxygenase from Agrobacterium tumefaciens (PDB code: 2I7G, amino acid sequence identity of 24\%) as search model. Automated model building was carried out with Buccaneer [12], and manual model building was performed in COOT [13]. Refinement of the initial electron density was carried out using cycles of the program phenix.refine [14]. The final structure was refined to $R_{\text {cryst }}=18 \%$ and $R_{\text {free }}=20 \%$ at resolution of $1.60 \AA$. The quality of the structure was validated by MOLPROBITY [15]. (Table S3).

\section{Crystallization and Structure Determination of RsI04}

After size exclusion chromatography (HiLoad Superdex S75 26/60, Merck, Darmstadt, Germany) in buffer solution (Tris/ $\mathrm{HCl}$ (pH 8.0) $20 \mathrm{~mm}, \mathrm{NaCl} 150 \mathrm{~mm}$ ), the protein was diluted to $20 \mathrm{mg} / \mathrm{ml}$. Initial screens were set up using the sitting drop vapor-diffusion method at $20^{\circ} \mathrm{C}$. A drop of $0.6 \mu \mathrm{L}$ total volume with different protein to reservoir ratios $(1: 3,1: 1,3: 1)$ was set with an automated crystallization drop-setter (Oryx Nano, Douglas Instrument, Berkshire, UK) and equilibrated against different reservoir solutions $(50 \mu \mathrm{L})$. Large single crystals appeared within two days with a reservoir condition containing $6 \%$ ethanol, $0.1 \mathrm{~m}$ ammonium acetate $(\mathrm{pH}$ 5.5) and $1.5 \mathrm{~m}$ phosphate ( $\mathrm{pH} 7.0)$. Crystals were mounted in nylon loops and flash-frozen in liquid nitrogen prior to data collection. A dataset to $1.8 \AA$ resolution was collected at beamline X06DA of the Swiss Light Source (Villigen, Switzerland) with a PILATUS $2 \mathrm{M}$ detector. The data were processed with autoPROC, and the crystal belonged to space group $P 3_{2} 21$, with unit cell dimensions of $a=b=83.7 \AA$ and $c=82.3 \AA$ [16]. The asymmetric unit contained two monomers, at a solvent content of $47.43 \%$. The crystal structure of RslO4 was determined by molecular replacement in MOLREP using the monomer of SnoaB (PDB: 3KNG, amino acid sequence identity of $41 \%$ ) as search model [17]. Refinement of the initial electron density was carried out by iteratively using phenix.refine, followed by model building in COOT $[13,14]$. The final structure was refined to $R_{\text {cryst }}=0.18$ and $R_{\text {free }}=0.20$ at a resolution of $1.8 \AA$. The geometry of the structure was validated by MOLPROBITY [15].

\section{In Vivo Comparison of RsIO4 and SnoaB Activities}

To compare between RslO4 and SnoaB enzymatic activity in the oxygenation of RslO4-substrate and reproducing rishirilide $\mathrm{B}$, each of encoding genes was cloned with replicative 
expression vector pUWL-H between ClaI and SpeI restriction sites and transferred into $S$. albus $\times \operatorname{Cos} 4 \Delta r s l O 4$ growing mycelia by intergeneric conjugation on MS-agar supported with $\mathrm{MgCl}_{2}$ (10 mM) using E. coli ET12567/ pUZ8002 cells. After $8 \mathrm{~h}$ incubation at $28^{\circ} \mathrm{C}$, the plates were overlaid with apramycin $(50 \mu \mathrm{g} / \mathrm{mL})$, hygromycin B $(50 \mu \mathrm{g} / \mathrm{mL})$, and fosfomycin $(200 \mu \mathrm{g} / \mathrm{mL})$ and incubated again at $28^{\circ} \mathrm{C}$ till the exconjugants appeared. The secondary metabolites were produced in $\mathrm{SG}+$ media at $28{ }^{\circ} \mathrm{C} / 180 \mathrm{rpm}$ for 5 days, then the crude extracts were extracted by ethyl acetate at $\mathrm{pH} 4$ and analyzed by HPLC/ESI-MS (table S2).

\section{Side-Directed Mutagenesis (SDM) Inside the Binding Cavity of RsI04 Protein}

The whole pUWL-H-rslO4 plasmid was PCR-amplified using back-to-back $5^{\prime}$-phosphorylated primers. At $5^{\prime}$-end, forward primers were designed with alanine codon for the substitution mutation of asparagine 7 , asparagine 52 or methionine 29 , and with phenylalanine codon for the substitution mutation of tryptophan 56 or tyrosine 22 (Table S1). In order to investigate the role of each amino acid in the binding to RslO4 substrate, the PCR products were selfligated and transferred separately into S. albus $\times \operatorname{Cos} 4 \Delta r s l O 4$ strain using the protocol of intergeneric conjugation on MSagar supported with $\mathrm{MgCl}_{2}(10 \mathrm{mM})$ using E. coli ET12567/ pUZ8002 cells. After $8 \mathrm{~h}$ incubation at $28{ }^{\circ} \mathrm{C}$, the plates were overlaid with apramycin $(50 \mu \mathrm{g} / \mathrm{mL})$, hygromycin B $(50 \mu \mathrm{g} / \mathrm{mL})$ and fosfomycin $(200 \mu \mathrm{g} / \mathrm{mL})$ and incubated again at $28^{\circ} \mathrm{C}$ till the exconjugants appeared. The secondary metabolites were produced in $\mathrm{SG}+$ media at $28^{\circ} \mathrm{C} / 180 \mathrm{rpm}$ for 5 days, then the crude extracts were extracted by ethyl acetate at $\mathrm{pH} 4$ and analyzed by HPLC/ESI-MS (Table S2). The residual activity of Rs1O4 was measured depending on calculating the relative AUC of reproduced rishirilide B in the crude extracts of $S$. albus $\times \operatorname{Cos} 4 \Delta$ rslO4 complemented with mutants Rs1O4 in Asn7, Asn52, Trp56, Tyr22 and Met29 comparing to relative AUC of reproduced rishirilide $\mathrm{B}$ in the crude extract of $\mathrm{S}$. albus $\times \mathrm{Cos} 4 \mathrm{ArslO} 4$ complemented with wild type RslO4.

\section{In Silico Prediction of RsI04-Ligands Interactions}

Molecular modelling was performed using the Schrödinger Software Suite (Schrödinger Release 2019-2). The structure of RslO4 was prepared using the Protein Preparation Wizard. In this step, atom types and bond orders were assigned, missing atoms were added, water molecules beyond 5 angstroms from hetero groups were deleted, water orientations were sampled, tautomer/ionization states were assigned corresponding to $\mathrm{pH} \mathrm{7,} \mathrm{the} \mathrm{hydrogen} \mathrm{bonding} \mathrm{network} \mathrm{was}$ optimized, followed by restrained minimization using the OPLS3 force field. In the next step, all putative substrates were docked into the active site of RslO4 using Glide XP with flexible ligand sampling. All default settings were used except intramolecular hydrogen bonds were rewarded and planarity of conjugated pi groups was enhanced.

The molecular dynamics simulations for selected models were performed using the GPU-accelerated Desmond program within the Schrödinger Software Suite (Schrödinger Release 2019-2). The SPC solvent model was used, and the force field was set to OPLS3e. The system energy was set to 1.2, and NPT was used as the ensemble class. All systems were relaxed before simulations. Simulations were set to run at $300.0 \mathrm{~K}$ and at 1.01325 bar. Simulation time for the putative substrate was set to $35 \mathrm{~ns}$ with $35 \mathrm{ps}$ trajectory recording intervals, and simulation time for the putative intermediate was set to $70 \mathrm{~ns}$ with $70 \mathrm{ps}$ trajectory recording intervals, resulting in an MD trajectory with 1000 frames per simulation.

\section{Results}

\section{Sequence Analysis of RsIC1, RsIC2, and RsIC3 Proteins}

Sequence analysis using BLASTp was used to predict the functions of RslC1, RslC2, and RslC3 proteins. First, the putative first ring CYC/ARO of nogalomycin (SnoaE), rhodomycin $(\mathrm{RdmK})$ and chartereusin $(\mathrm{ChaF})$ were found to be homologs of RslC1 with 53\%, 54\% and 55\% identity, respectively $[18,19]$. Second, the homologs of RslC2 included ActIV (55\% identity), AlnR (54\%) and Ssfy2 (53\%) that act probably as second ring CYC/ARO in the biosynthesis of actinorhodin, alnumycin and tetracycline SF2575, respectively [20-22]. Third, sequence alignments of RslC3 to the proposed fourth ring CYC/ARO in chromomycin $(\mathrm{CmmX})$, oxytetracycline (OxyL) and nogalomycin (SnoO) showed $49 \%, 44 \%$ and $50 \%$ identity, respectively [23-25].

\section{Inactivation of rs/C3 and rs/O4 in S. bottropensis}

In order to show that $r s l C 3$ is involved in rishirilide biosynthesis and in order to confirm the involvement of $\mathrm{rslO}^{\mathrm{O}}$ gene deletion experiments were carried out in S. bottropensis. Trace amounts of rishirilide were detected in the crude extract of $S$. bottropensis $\Delta$ rslC3. In addition, the tricyclic aromatic galvaquinones (Fig. S1) could be detected in the rslC3 mutant (galvaquinone 1 and 2). Complementation of the $r s l C 3$ mutant led to an increase in rishirilide $\mathrm{B}$ production but formation of galvaquinones was also observed (Fig. $\mathrm{S} 1$ ), indicating that the biosynthetic machinery in the complemented mutant did not work as well as in the wt-strain. $S$. bottropensis with a deletion in $\mathrm{rslO}^{\mathrm{O}} 4$ also failed to produce rishirilides but accumulated galvaquinone 1 and 2 instead. 
In this mutant, galvaquinones production increased when a plasmid containing the positive regulatory genes $r s l R l$, $r s l R 2$ and $r s l R 3$ were coexpressed (Fig. S2) resulting in a higher transcription of structural genes. The galvaquinones 1 and 2 were identical in their HPLC-retention times, UV- and Mass-spectra compared to galvaquinones A and B respectively. Structures of both compounds have been elucidated before as products of S. albus $\times$ Cos4 4 rslO4 [5] (Supplementary Fig. S3). Based on UV- and MS spectra Galvaquinone 2 is identical to Galvaquinone $\mathrm{B}$ but its retention time in HPLC is different.

\section{Molecular Weight and Stability of RsI01 and RsI04}

According to SDS-PAGE analysis, the molecular weights of C-terminal his-tagged proteins $\mathrm{RslO} 1$ and $\mathrm{Rs} 1 \mathrm{O} 4$ were consistent with the predicted ones: 40.04 and $12.17 \mathrm{kDa}$, respectively. After $12 \mathrm{~h}$ on ice, no precipitation was observed, in addition to the stability by melting from freezing condition at $-80^{\circ} \mathrm{C}$ to the room temperature.

\section{Crystal Structure of RsIO4 (PDB ID:7BIO)}

The monooxygenase RslO4 crystallizes as a homodimer and the overall structure of each subunit is made up of three $\alpha$-helices $(\alpha 1-\alpha 3)$ and four $\beta$-strands $(\beta 1-\beta 4)$ with a ferredoxin-like fold (Fig. 1a). The additional $\alpha$-helix in the $\mathrm{C}$-terminus of each monomer is generated by the TEV protease cleavage site of the His-tag sequence, which is required for protein purification. The three $\alpha$-helices are located on one side of the homodimer. Together with an additional $\beta$-strand of the other subunit, a five-stranded antiparallel $\beta$-sheet (Fig. 1b-d), which is stabilized by ten main-chain hydrogen bonds between $\beta 2$ and $\beta 4$ of two chains. This strand-swapping feature in dimer formation and stabilization can also be observed in homologous structures such as the monooxygenases ActVA-Orf6 from Streptomyces coelicolor and SnoaB from Streptomyces nogalater.

The interface between the two monomers is further stabilized by hydrophobic interactions provided by $\beta$-sheets and side chain hydrogen bonds contributed by Lys8, Ser43, Glu85 and His87. Data collection and refinement statistics are shown in table S3.

The closest structural homologue is SnoaB from $S$. nogalater, with a sequence identity of $41 \%$. The second closest homologue, with an identity of $28 \%$ is SL3150, a monooxygenase from Shewanella loihica involved in antibiotic biosynthesis. All the homologues share a similar fold, even though the sequence identities for some of them are lower than $10 \%$. The homologues are mostly uncharacterized and annotated as putative monooxygenases. Only a few have been biochemically investigated, including ActVA-Orf6 (of $16 \%$ identity), SnoaB and MhuD (of $22 \%$ identity). MhuD is a heme-degrading monooxygenase from Mycobacterium tuberculosis. It binds two heme groups, indicating the high flexibility of the substrate binding pocket. Sequence alignment of RslO4 together with the monooxygenases SnoaB and ActVA-Orf6, which both convert phenolic substrates to quinone products, showed that Asn52 and Trp56 are conserved in the negatively-charged active site (Fig. 2).

Closer inspection of the active site of $\mathrm{RslO} 4$ revealed high similarity between the positioning of Asn7, Asn52 and Trp56,
Fig. 1 RslO4 Crystal structure: a Subunit A of RslO4 monooxygenase with the secondary structure elements labelled. The monomer contains three $\alpha$ helices $(\alpha 1-\alpha 3)$ and four $\beta$ strands $(\beta 1-\beta 4)$ with a ferredoxin fold. The dimeric apo-form of $\mathrm{Rs} 1 \mathrm{O} 4$ is shown from the side (b) and top view (c). The two subunits are coloured in green and blue, respectively. (d) Electrostatic potential surface shows a positively charged substrate binding pocket (SBP)
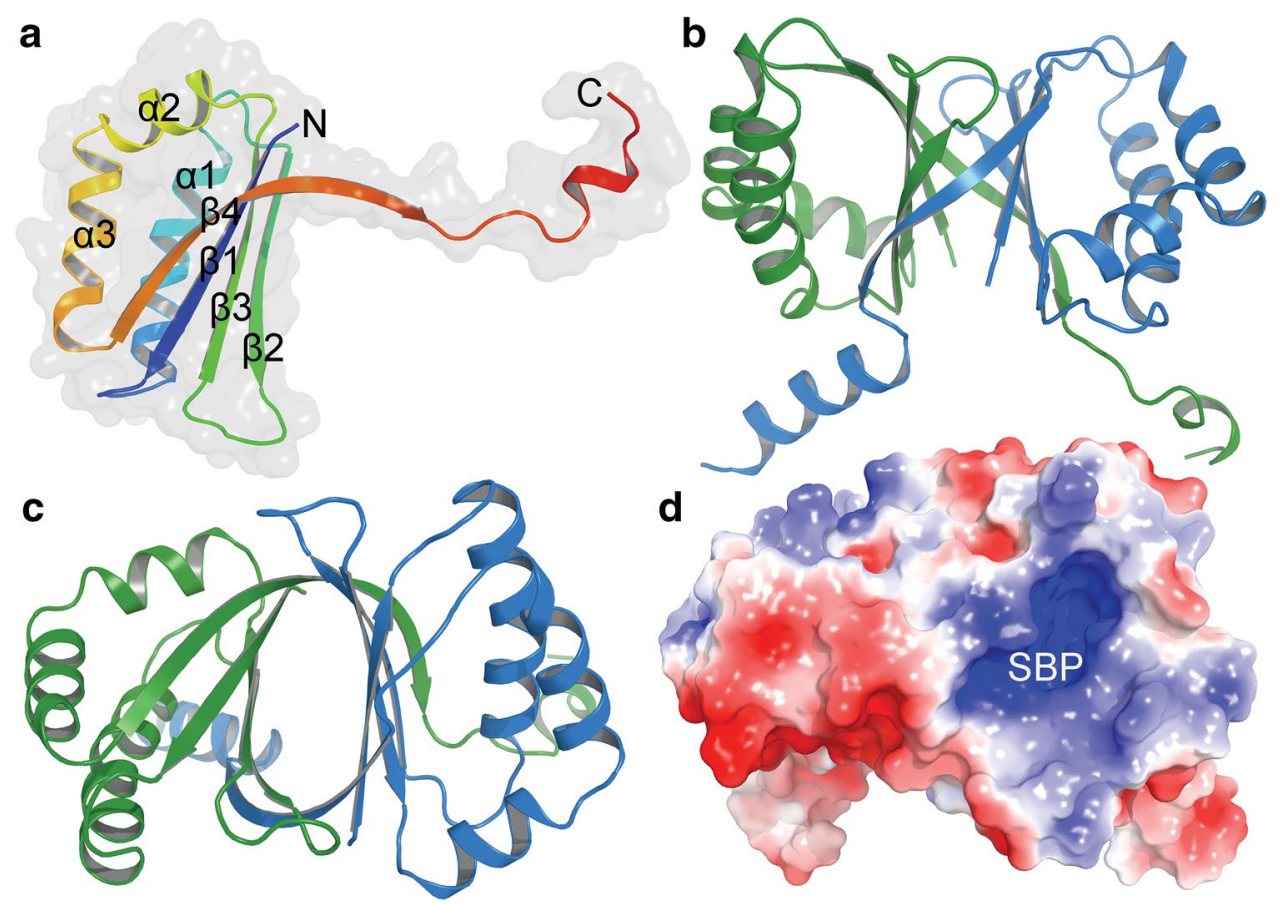


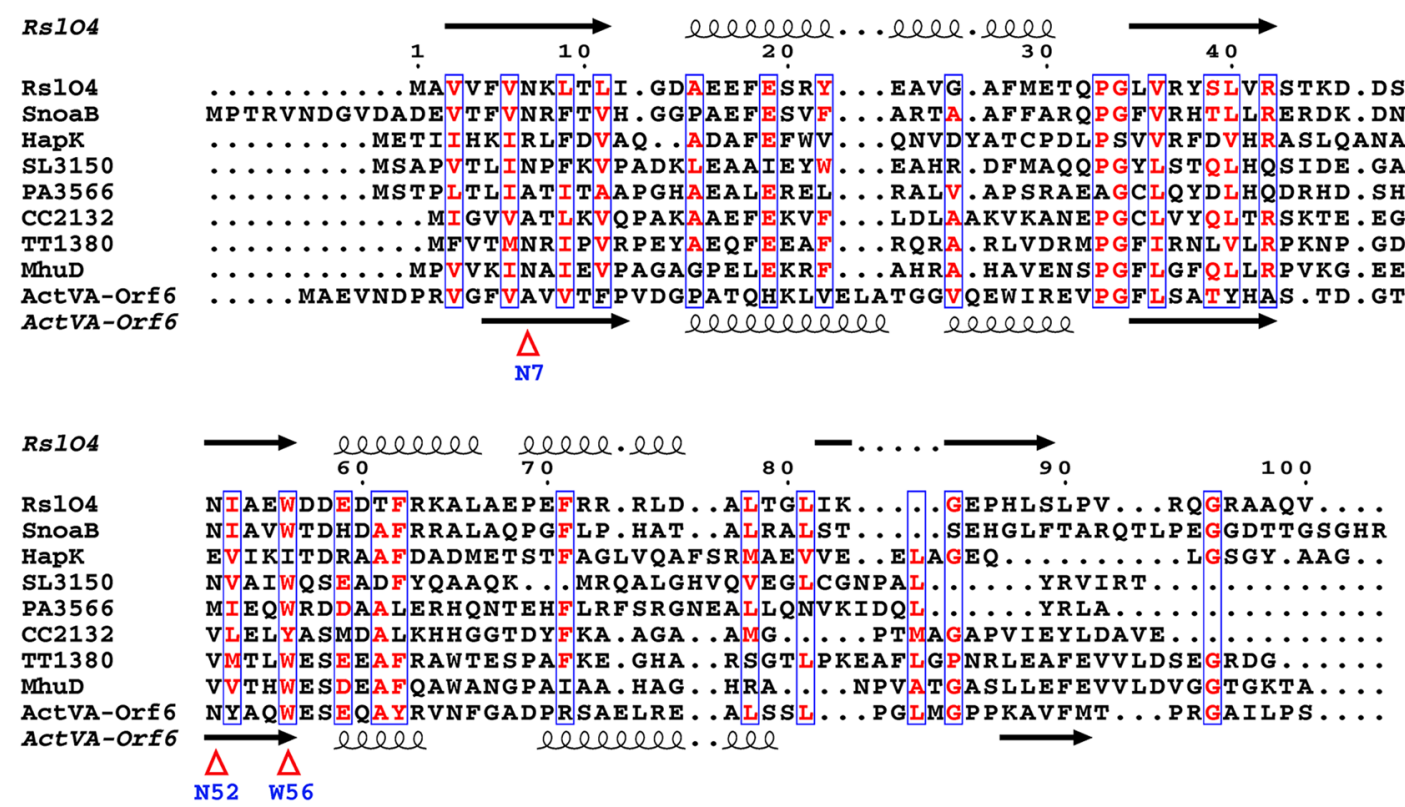

Fig. 2 Sequence alignment of monooxygenases with the same fold like Rs1O4: The sequences have been selected based on identity from the output of SWISS-MODEL search, and the overall sequence identities between RslO4 and homologies are ranging from 16 to $41 \%$ [26]. SnoaB (UniProtKB AC: O54259) is a cofactor-independent monooxygenase from $S$. nogalater (PDB code: 3KNG) [27]. HapK (Q2S9J0) is a RedY-like protein from Hahella chejuensis KCTC 2396 (2JDJ) [28]. SL3150 (A3QHR8) is an antibiotic biosynthesis monooxygenase from Shewanella loihica (2RIL). PA3566 (Q9HY51)

which are represented by Asn18, Asn63 and Trp67 in SnoaB coordinating oxidized dithranol by hydrogen bonds. Comparing to ActVA-Orf6, positioning of Asn52 and Trp56, which are represented by Asn62 and Trp66 in ActVA-Orf6 coordinating oxidized acetyl dithranol by hydrogen bonds showed also high similarity. The hydrogen bond formed by Tyr51 in ActVA-Orf6 could be replaced by Tyr22 in RslO4.

Observation from $35 \mathrm{~ns}$ molecular dynamic simulations suggested that the natural substrate of $\mathrm{RslO} 4$ is composed of tricyclic aromatic triol-anthracene structure with methyl group and methyl pentyl sidechain on the third aromatic ring. The Trp56 initiates a hydrogen bonding network to the three -OH groups (Fig. 3). On the hydrophobic side, Asn7-Asn52Wat 3 triad located close to the third aromatic ring. In $70 \mathrm{~ns}$ molecular dynamics simulations, this triad forms a hydrogen bond with the peroxide in the proposed intermediate of $\mathrm{RslO} 4$ (Fig. 4). It was also observed that Tyr22 contributes in the stabilization of peroxy intermediate inside the binding pocket.

\section{In Vivo Comparison of RsIO4 and SnoaB Enzymatic Activities}

In order to compare the monooxygenase activity of RslO4 with SnoaB, $S$. albus $\times$ Cos $4 \Delta r s l O 4$ was transformed with is an uncharacterized protein from Pseudomonas aeruginosa PAO1 $(1 \mathrm{X} 7 \mathrm{~V})$ [29]. CC2132(Q9A6G2) is an uncharacterized protein from Caulobacter vibrioides ATCC 19089 (3BM7). TT1380 (P83693) is from Thermus thermophiles HB8 (1IUJ) [30]. MhuD (P9WKH3) is a heme-degrading monooxygenase from Mycobacterium tuberculosis (3HX9) [31]. ActVA-Orf6 (Q53908) is a monooxygenase involved in the actinorhodin biosynthesis from S. coelicolor (1N5T) [32]. Sequence alignment was performed in ClustalW and the figure was prepared by ESPript 3.0 server [33, 34]

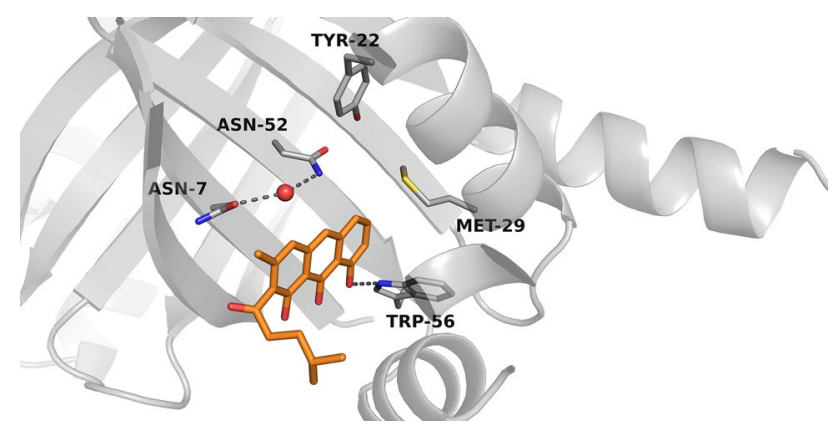

Fig. 3 Predicted binding pose of the proposed tricyclic aromatic substrate inside RslO4 binding site

each of $r s l O 4$ and $s n o a B$ genes cloned with replicative vector pUWL-H to test their enzymatic abilities in RslO4substrate oxygenation and reproduce rishirilide B. Rishirilide B was successfully reproduced by complementation with rslO4. In contrast, no production of rishirilide B could be observed in $S$. albus $\times \operatorname{Cos} 4 \Delta$ snoaB . 


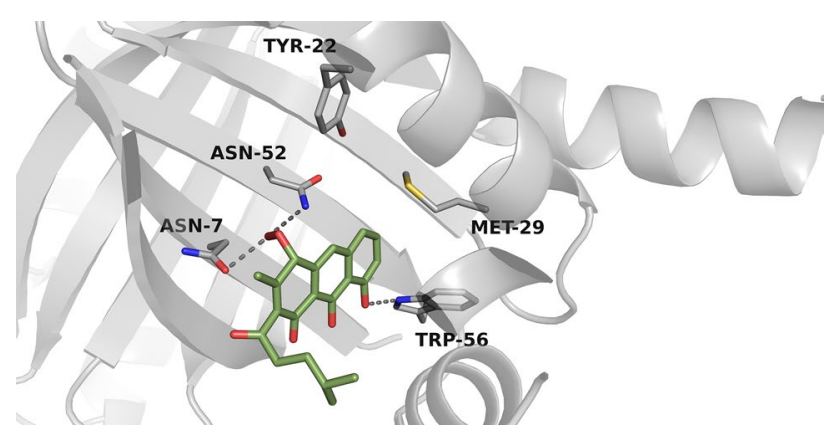

Fig. 4 Predicted binding pose of the proposed peroxy intermediate inside RslO4 binding site

\section{Investigation on Oxygenation Mechanism of RsIO4 by Site-Directed Mutagenesis}

For deeper inspection of RslO4 catalytic machinery, substitution mutations in specific residues, which form the binding center, were created. Asparagine7, asparagine52 and methionine 29 were replaced with alanine, while tryptophan56 and tyrosine 22 were replaced with phenylalanine. Changes in rslO4 gene sequence were performed in pUWL-H vector, thus it was possible to detect the incorporation of each residue in the enzymatic activity in rishirilide B biosynthesis in $S$. albus $\times$ Cos $4 \Delta$ rslO4. These results revealed that there is a clear trend of decreasing in rishirilide $\mathrm{B}$ production by the complementation of $\mathrm{S}$. albus $\times \mathrm{Cos} 4 \mathrm{\Delta rslO} 4$ with sitespecific mutated $\mathrm{rslO}_{4}$ genes comparing to the wild type. It was found that the substitution mutation of each of asparagine 7 and asparagine 52 reduced the production of rishirilide B by approx. $92 \%$, whereas the replacement of tryptophan56 resulted in approx. $60 \%$ reduction. Creating substitution mutations of tyrosine 22 and methionine 29 showed the lowest influence on rishirilide B production (approx. 40\% reduction in the activity) (Fig. S4).

\section{Crystal Structure of RsI01 (PDB ID: 7BIP)}

The monooxygenase Rs1O1 crystallizes as a homodimer and the overall structure of each subunit is made up of a well-conserved $8 \beta$-strands TIM-barrel surrounded by $8 \alpha$-helixes. Each $\beta$-strand connects an $\alpha$-helix through a short loop region. According to the structural homology study using Swiss Model Server, RslO1 was revealed to be FMN-dependent bacterial luciferase-like monooxygenase (Fig. 5). Data collection and refinement statistics are shown in table S3.

Structural homologs for RslO1 include MoxC (UnitprotKB AC: O34974) a putative monooxygenase from $B$. subtilis (PDB code: 1YW1); Lad A (A4IU28) an alkane monooxygenase from G.thermodenitrificans (3B9O) [35]; MeR (Q8TXY4) a 5,10-methylenetetrahydromethanopterin reductase from $M$. kandleri (1EZW) [36]; FgD1 (P9WNE1) a F420-dependent glucose-6-phosphate dehydrogenase from M. tuberculosis (3C8N) [37, 38]; AdF (O93734) a F420-dependent alcohol dehydrogenase from $M$. thermophiles (1RHC) [39]; SsuD (P80645) an alkanesulfonate
Fig. 5 RslO1 crystal structure: a Stereo view of apo-form monooxygenase RslO1 structure. The two monomers form a tight homodimer. $\mathbf{b}$ In subunit A, the substrate binding site was occupied by a polyethylene glycol (PEG) molecule derived from the crystallization condition. c Electrostatic potential surface shows positively charged substrate binding pocket (SBP) a
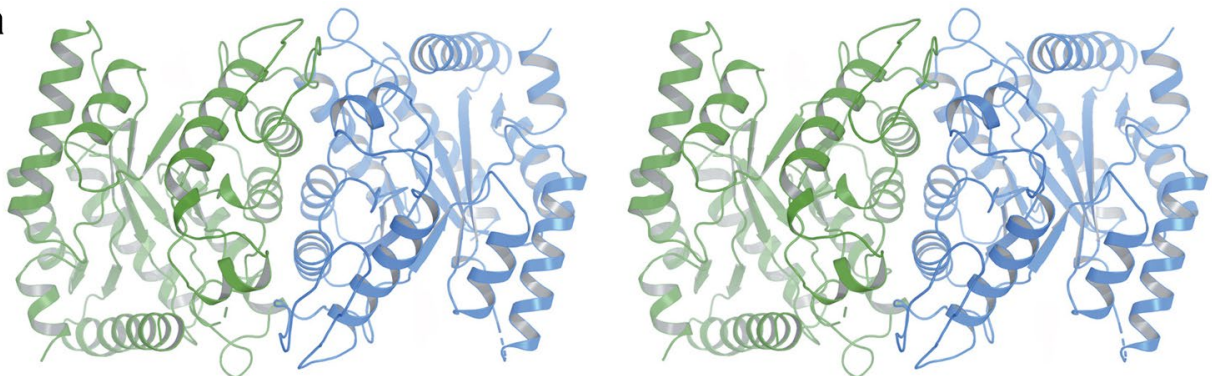

\section{с}
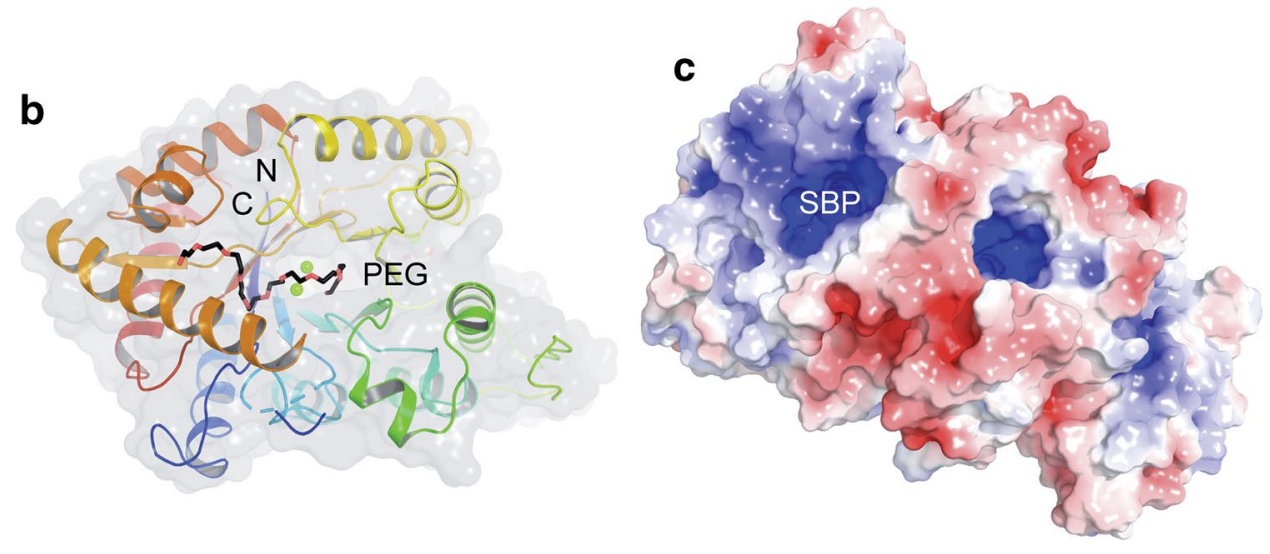
monooxygenase from E. coli (1M41) [40]; LuxA (P07740) an alpha chain of an alkanal monooxygenase from V.harveyi (3FGC) [41]; and MsnO8 (W8QCH3), a luciferase-like monooxygenase from $S$. bottropensis (4US5) [42].

Protein sequence alignment of $\mathrm{RslO} 2$ with $\mathrm{MsnO} 3$ revealed a high identity (47\%) using BLASTp. MsnO3 acts as flavin reductase by NADH to provide MsnO8 with the essential reduced FMN for epoxidation and conversion of deoxymensacarcin to mensacarcin [42]. A summary of results obtained in this study is given in Fig. 6 .

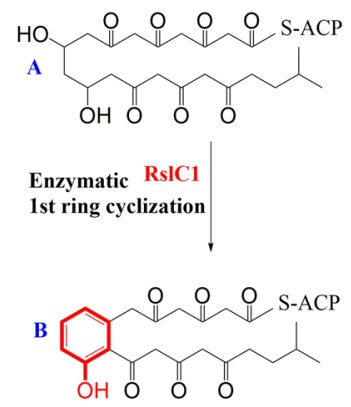

\section{Discussion}

In our previous studies about rishirilide biosynthesis we could show that RslK4 and RslO3 are involved in the selection of the starter unit. The assembly of rishirilide polyketone from 9 acetate units and one valine-derived isobutyrate is mediated by the minimal PKSs (RslK1, RslK2, RslK3) and RslA [4, 5]. It was proposed that RslO10 acts as C9-ketoreductase and supports the first ring cyclization in rishirilide polyketone [5].

Sequence analysis of $r s l C 1, r s l C 2$ and $r s l C 3$ suggests that they act as cyclases during rishirilide biosynthesis. Homology of RslC1 (SnoaE 53\%, RdmK 54\% and ChaF $55 \%$ ) is responsible for 1 st ring cyclization in nogalomycin-, rhodomycin- and chartereusin-biosynthesis,
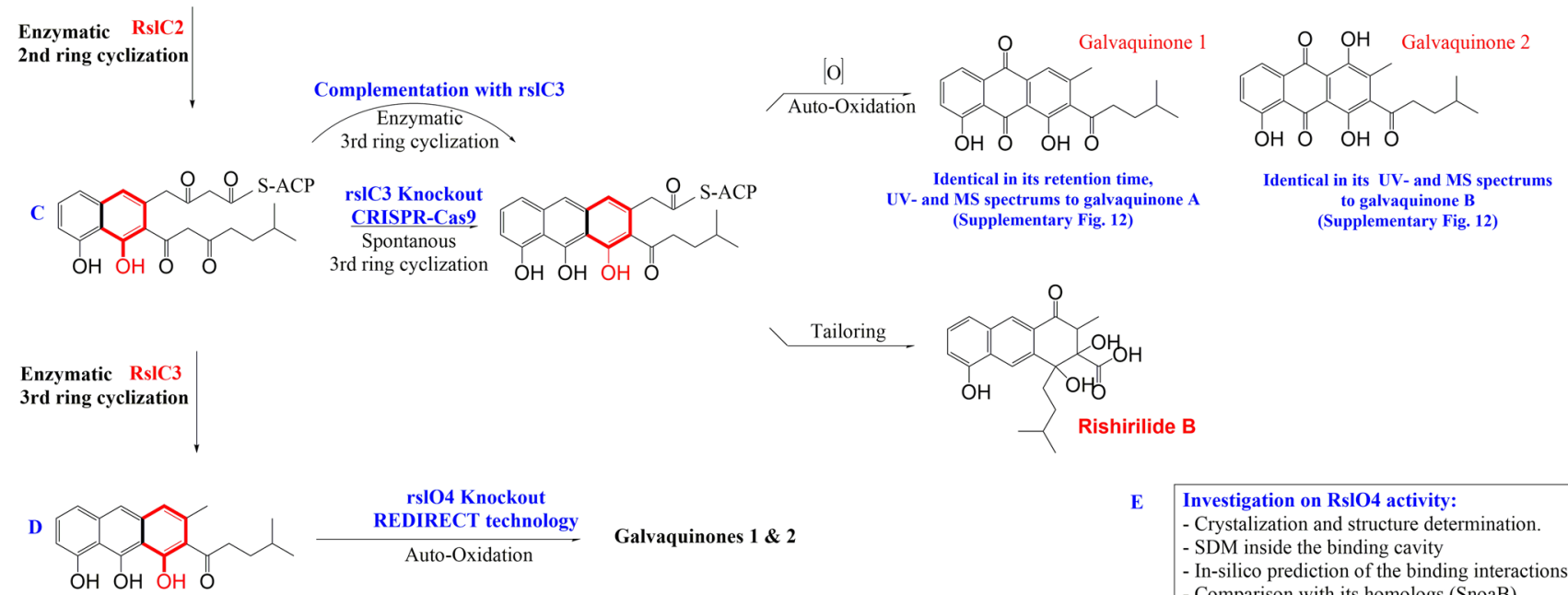

E Investigation on RslO4 activity: - Crystalization and structure determination. - SDM inside the binding cavity - In-silico prediction of the binding interactions - Comparison with its homologs (SnoaB)

F Investigation on RslO1/RsIO2 activity: - Crystalization of RslO1

- Comparison with RslO1 homologs (MsnO8) - Comparison with RslO2 homolog (MsnO3)

Fig. 6 Flow chart of methods and results obtained in this study: a Based on sequence comparison RslC1 is responsible for the enzymatic first ring cyclization/aromatization in rishirilide biosynthesis and b RslC2 is responsible for the enzymatic second ring cyclization/aromatization in rishirilide biosynthesis. c Knockout of 3rd ring cyclase gene "rslC3" using CRISPR-Cas9 system resulted in the pro- duction of small amounts of galvaquinone 1 and 2. Complementation with $r s l C 3$ gene resulted in the reproduction of rishirilide B. d Knockout of the monooxygenase "rslO4" using REDIRECT® technology resulted in the production of small amount of galvaquinones 1 and 2. e Studies on RslO4. f Studies on RslO1/RslO2 
respectively $[18,19]$. We therefore propose that $\mathrm{RslC} 1$ and Rs1O10 mediate the cyclization/aromatization of the 1 st ring in rishirilide-biosynthesis. RsIC2 homologs (ActIV 55\%, AlnR 54\% and Ssfy2 53\%) act as 2nd ring cyclases during actinorhodin-, alnumycin- and tetracycline-biosynthesis, respectively [20-22]. Thus RslC2 is considered as 2nd cyclase in rishirilide biosynthesis. And homologs of RslC3 (Cmm 49\%, Oxy 44\% and SnoO 50\%) are involved in ring cyclization of the last ring during the biosynthesis of chromomycin A3, oxytetracycline and nogalomycin, respectively. Thus RslC 3 is most likely the $3^{\text {rd }}$ ring cyclase involved in rishirilide biosynthesis [23-25].

A mutant with a deletion in $r s l C 3$ was still producing small amount of rishirilide B indicating that the formation of the third ring can also occur non-enzymatically.

The production of galvaquinone 1 (galvaquinone $\mathrm{A}$ ) and galvaquinone 2 in our mutants indicate that the cyclization step 3, catalyzed by RslC3, also occurs chemically, although to a much smaller extend. It also indicates that early intermediates of the pathway chemically easily convert to Galvaquinone derivatives. (Supplementary Fig. S1).

The deep-sea-derived S. olivaceus SCSIO T05 was described to produce rishirilides and galvaquinone derivatives. The rishirilide cluster (rsd-cluster) in this strain is very similar to the rsl-cluster in S. bottropensis. [2]. And recently the rishirilide derivative Lupinacidin A and Galvaquinone $B$ have been isolated from the sea anemone Gyractis sesere [43]. These results indicate that intermediates of biosynthetic pathways to rishirilides can chemically be converted efficiently to galvaquinones.

As we were not able to detect novel intermediates of the pathway in our mutants we decided to focus our research on RslO4 and RslO1, which are both involved in biosynthetic steps which follow the cyclization process.

The high similarity of RslO4 in the sequence and threedimensional structure with the monooxygenase SnoaB suggests that RslO4 mediates a mono-oxygenation in rishirilide biosynthetic pathway. Interestingly, complementation of $S$. albus $\times \mathrm{Cos} 4 \mathrm{AslO} 4$ with $r s l O 4$ restored rishirilide production but complementation with snoaB did not indicate different function of both enzymes. Unlike SnoaB which oxygenates the middle non-aromatic ring of 12-deoxynogalonic acid during nogalomycin biosynthesis [27], RslO4 might oxygenate the third lateral aromatic ring in its tricyclic aromatic substrate to form a quinonic product.

According to the observation from molecular dynamics simulations, Trp56 contributes in the orientation of substrate positioning inside $\mathrm{Rs} \mathrm{OO} 4$ pocket through an $\mathrm{H}$-Bonding network with the three - $\mathrm{OH}$ groups, which in turn leads to an electron delocalization in the third aromatic ring. It is possible that Asn7-Asn52-Wat3 triad catalyzes a proton abstraction from the tricyclic aromatic substrate and reduces the activated peroxide with the same proton to form a peroxy intermediate (Fig. 7).

This intermediate is stabilized inside RslO4 binding center by Asn7-Asn52-Wat3 triad and Trp56, in addition to Tyr22 through hydrogen bindings. The results of dynamic simulations match those of the Site-Directed Mutagenesis which demonstrated the importance of Asn7, Asn52, Trp56, Tyr22, and Met29 in the catalytic mechanism of RslO4. Although the computer simulations did not indicate any cooperation of Met29 in the reaction, it is conceivable that this residue helps in the protein conformational stability.

The present mechanism seems to be consistent with other research which found that Trp67 in SnoaB and Trp66 in ActVA-Orf6 are mainly responsible for the orientation and electron delocalization in their substrates. There are similarities between the roles of Asn7-Asn52-Wat3 triad in RslO4 and the role of Asn18-Asn63-Wat33 in SnoaB. In accordance with the contribution of Tyr51 in stabilizing the peroxy intermediate of ActVA-Orf6, Tyr22 seems to be important for the stabilization of RslO4 intermediate. Following oxygenation, this intermediate would release $\mathrm{H}_{2} \mathrm{O}$ to form RslO4 quinone product [27, 32, 44] (Fig. 8).

RslO1 belongs to the family of FMN-dependent luciferase-like monooxygenases. The high similarity in threedimensional structure between RslO1 and the epoxyforming MsnO8 enzyme in mensacarcin biosynthesis in $S$. bottropensis indicates a similar activity of RslO1 enzyme in the epoxide formation to form RslO5-substrate during rishirilide biosynthesis in the same strain. According to the high sequence identity between $\mathrm{RslO} 2$ and $\mathrm{MsnO} 3$ $(47 \%)$, it is most likely that $\mathrm{RslO} 2$ acts as flavin reductase, by using NADH as a hydrogen source, to provide RslO1 with the reduced FMN in a similar mechanism of

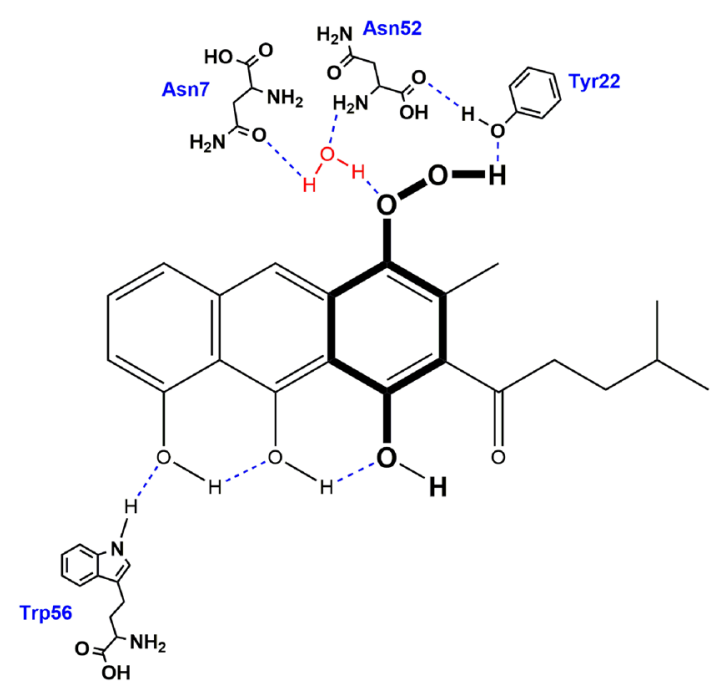

Fig. 7 Proposed binding of a putative substrate in the binding pocket of Rs1O4 

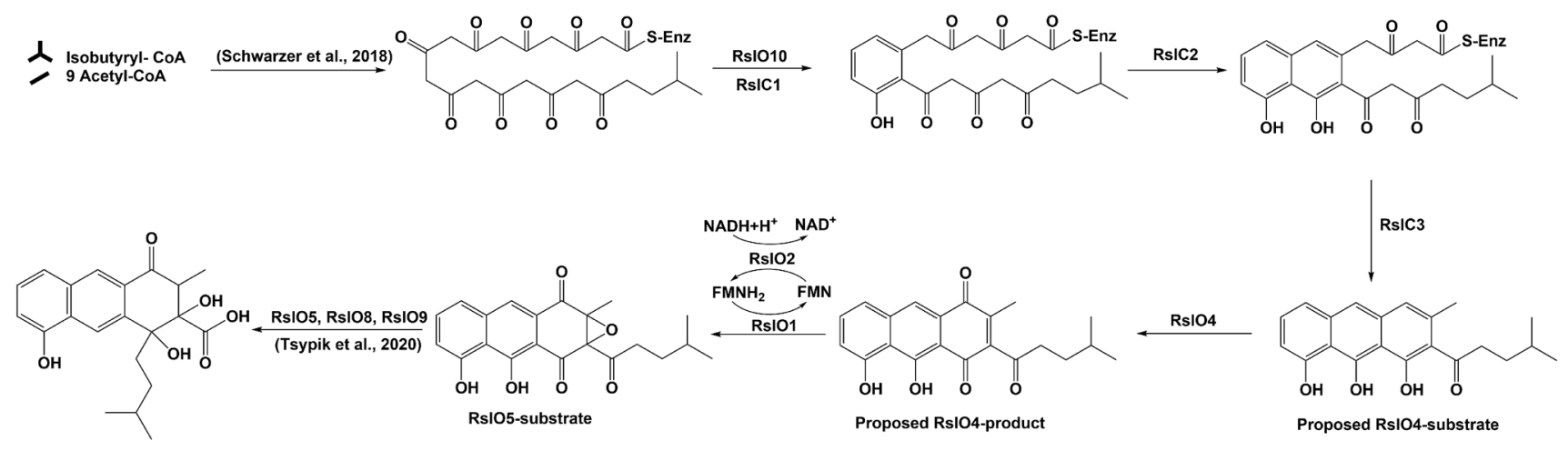

Fig. 8 Proposed Biosynthetic pathway of rishirilide B

MsnO8-MsnO3 two-component epoxide-forming system [42] (Fig. 8).

Following the epoxidation mediated by RslO1-RslO2 system, Rs1O1 product undergoes an epoxide reduction by Rs1O5 and then oxidative carbon backbone rearrangement including Rs1O9-mediated Baeyer-Villiger oxidation and lactone formation. Subsequently, Rs1O8 catalyzes a keto-reduction to form rishirilide A. Lactone-opening and water elimination in rishirilide A leads to the production of rishirilide B [6] (Fig. 8).

\section{Conclusion}

During rishirilide biosynthesis, RslC3 mediates a cyclization step leading to an intermediate with an unknown structure. The cofactor-independent monooxygenase RslO4 catalyzes the quinone formation of the third ring. The putative flavin reductase $\mathrm{Rs} 1 \mathrm{O} 2$ uses NADH as a source of hydrogen and provides RslO1 with the reduced FMN. In his turn the FMN-dependent luciferase-like monooxygenase RslO1 is responsible for the formation of an epoxide providing a substrate for RsiO5.

Supplementary Information The online version contains supplementary material available at https://doi.org/10.1007/s12033-021-00314-x.

Funding Open Access funding enabled and organized by Projekt DEAL. This study was funded by Deutsche Forschungsgemeinschaft (Grant No. RTG 1976).

\section{Declarations}

Conflict of interest The authors declare no conflict of interest related to this study.

Open Access This article is licensed under a Creative Commons Attribution 4.0 International License, which permits use, sharing, adaptation, distribution and reproduction in any medium or format, as long as you give appropriate credit to the original author(s) and the source, provide a link to the Creative Commons licence, and indicate if changes were made. The images or other third party material in this article are included in the article's Creative Commons licence, unless indicated otherwise in a credit line to the material. If material is not included in the article's Creative Commons licence and your intended use is not permitted by statutory regulation or exceeds the permitted use, you will need to obtain permission directly from the copyright holder. To view a copy of this licence, visit http://creativecommons.org/licenses/by/4.0/.

\section{References}

1. Iwaki, H., Nakayama, Y., Takahashi, M., Uetsuki, S., Kido, M., \& Fukuyama, Y. (1984). Structures of rishirilides A and B, $\alpha 2$-macroglobulin inhibitors produced by Streptomyces rishiriensis OFR-1056. Journal of Antibiotics, 37(9), 1091-1093.

2. Zhang, C., Sun, C., Huang, H., Gui, C., Wang, L., Li, Q., \& Ju, J. (2018). Biosynthetic baeyer-villiger chemistry enables access to two anthracene scaffolds from a single gene cluster in deep-seaderived Streptomyces olivaceus SCSIO T05. Journal of Natural Products, 81(7), 1570-1577.

3. Odagi, M., Furukari, K., Takayama, K., Noguchi, K., \& Nagasawa, K. (2017). Total synthesis of rishirilide B by organocatalytic oxidative kinetic resolution: Revision of absolute configuration of (+)-Rishirilide B. Angewandte Chemie International Edition, 56, 6609-6612.

4. Schwarzer, P., Wunsch-Palasis, J., Bechthold, A., \& Paululat, T. (2018). Biosynthesis of rishirilide B. Antibiotics, 7(1), 20.

5. Schwarzer, P., Tsypik, O., Zuo, C., Alali, A., Wunsch-Palasis, J., Heitzler, T., Derochefort, J., Bernhardt, M., Yan, X., Paululat, T., 
\& Bechthold, A. (2020). Early steps in the biosynthetic pathway of rishirilide B. Molecules, 25(8), 1955.

6. Tsypik, O., Makitrynskyy, R., Frensch, B., Zechel, D. L., Paululat, T., Teufel, R., \& Bechthold, A. (2020). Oxidative carbon backbone rearrangement in rishirilide biosynthesis. Journal of the American Chemical Society, 142(13), 5913-5917.

7. Tong, Y., Charusanti, P., Zhang, L., Weber, T., \& Lee, S. Y. (2015). CRISPR-Cas9 based engineering of actinomycetal genomes. ACS Synthetic Biology, 4(9), 1020-1029.

8. Gust, B., Kieser, T., \& Chater, K. F. (2002). REDIRECT@ technology: PCR-targeting system in Streptomyces coelicolor. Department of Molecular Microbiology, John Innes Centre, Norwich Research Park.

9. Battye, T. G. G., Kontogiannis, L., Johnson, O., Powell, H. R., \& Leslie, A. G. W. (2010). iMOSFLM: A new graphical interface for diffraction image processing with MOSFLM. Acta Cryst., 67, 271-281.

10. Kabsch, W. (2009). XDS. Acta Crystallographica, 66, 125-132.

11. Keegan, R. M., \& Winn, M. D. (2007). MrBUMP: an automated pipeline for molecular replacement. Acta Crystallographica, 64, 119-124.

12. Cowtan, K. (2006). The Buccaneer software for automated model building. 1. Tracing protein chains. Acta Crystallographica, 62, 1002-1011.

13. Emsley, P., \& Cowtan, K. (2004). Coot: Model-building tools for molecular graphics. Acta Crystallographica, 60, 2126-2132.

14. Afonine, P. V., Grosse-Kunstleve, R. W., Echols, N., Headd, J. J., Moriarty, N. W., Mustyakimov, M., Terwilliger, T. C., Urzhumtsev, A., Zwart, P. H., \& Adams, P. D. (2011). Towards automated crystallographic structure refinement with phenix. refine. Acta Crystallographica, 68, 352-367.

15. Chen, V. B., Arendall, W. B., III., Headd, J. J., Keedy, D. A., Immormino, R. M., Kapral, G. J., Murray, L. W., Richardson, J. S., \& Richardson, D. C. (2009). MolProbity: All-atom structure validation for macromolecular crystallography. Acta Crystallographica, 66, 12-21.

16. Vonrhein, C., Flensburg, C., Keller, P., Sharff, A., Smart, O., Paciorek, W., Womack, T., \& Bricogne, G. (2010). Data processing and analysis with the autoPROC toolbox. Acta Crystallographica, 67, 293-302.

17. Vagin, A., \& Teplyakov, A. (2009). Molecular replacement with MOLREP. Acta Crystallographica, 66, 22-25.

18. Xu, Z., Jakobi, K., Welzel, K., \& Hertweck, C. (2005). Biosynthesis of the antitumor agent chartreusin involves the oxidative rearrangement of an anthracyclic polyketide. Chemistry \& Biology, 12(5), 579-588.

19. Klymyshin, D. A., Stefanyshyn, O. N., \& Fedorenko, V. A. (2015). Role of genes snoaM, snoaL, and snoaE in the biosynthesis of nogalamycin in Streptomyces nogalater Lv65. Cytology and Genetics, 49, 152-157.

20. Taguchi, T., Awakawa, T., Nishihara, Y., Kawamura, M., Ohnishi, Y., \& Ichinose, K. (2017). Bifunctionality of ActIV as a cyclase-thioesterase revealed by in vitro reconstitution of actinorhodin biosynthesis in Streptomyces coelicolor A3(2). ChemBioChem, 18(3), 316-323.

21. Oja, T., Palmu, K., Lehmussola, H., Leppäranta, O., Hännikäinen, K., Niemi, J., Mäntsälä, P., \& Metsä-Ketelä, M. (2008). Characterization of the alnumycin gene cluster reveals unusual gene products for pyran ring formation and dioxan biosynthesis. Chemistry \& Biology, 15(20), 1046-1057.

22. Pickens, L. B., Kim, W., Wang, P., Zhou, H., Watanabe, K., Gomi, S., \& Tang, Y. (2009). Biochemical analysis of the biosynthetic pathway of an anticancer tetracycline SF2575. Journal of the American Chemical Society, 131(48), 17677-17689.

23. Menendez, N., Nur-e-Alam, M., Brana, A. F., Rohr, J., Salas, J. A., \& Mendez, C. (2004). Biosynthesis of the antitumor chromomycin A3 in Streptomyces griseus: Analysis of the gene cluster and rational design of novel chromomycin analogs. Chemistry \& Biology, 11(1), 21-32.

24. Petković, H., Lukežič, T., \& Suš̌ković, J. (2017). Biosynthesis of oxytetracycline by Streptomyces rimosus: Past, present and future directions in the development of tetracycline antibiotics. Food Technology and Biotechnology, 55(1), 3-13.

25. Gullon, S., Olano, C., Abdelfattah, M. S., Brana, A. F., Rohr, J., Mendez, C., \& Salas, J. A. (2006). Isolation, characterization, and heterologous expression of the biosynthesis gene cluster for the antitumor anthracycline steffimycin. Applied and Environment Microbiology, 72(6), 4172-4183.

26. Waterhouse, A., Bertoni, M., Bienert, S., Studer, G., Tauriello, G., Gumienny, R., Heer, F. T., de Beer, T. A. P., Rempfer, C., Bordoli, L., Lepore, R., \& Schwede, T. (2018). SWISSMODEL: Homology modelling of protein structures and complexes. Nucleic Acids Research, 46(Web Server issue), 296-303.

27. Grocholski, T., Koskiniemi, H., Lindqvist, Y., Mäntsälä, P., Niemi, J., \& Schneider, G. (2010). Crystal structure of the cofactor-independent monooxygenase SnoaB from Streptomyces nogalater: Implications for the reaction mechanism. Biochemistry, 49(5), 934-944.

28. Rezacova, P., Borek, D., Moy, S. E., Joachimiak, A., \& Otwinowski, Z. (2008). Crystal structure and putative function of small Toprim domain-containing protein from Bacillus stearothermophilus. Proteins, 70(2), 311-319.

29. Sanders, D. A. R., Walker, J. R., Skarina, T., \& Savchenko, A. (2005). The X-ray crystal structure of PA3566 from Pseudomonas aureginosa at $1.8 \AA$ resolution. Proteins, 61, 209-212.

30. Wada, T., Shirouzu, M., Terada, T., Kamewari, Y., Park, S. Y., Tame, J. R. H., Kuramitsu, S., \& Yokoyama, S. (2004). Crystal structure of the conserved hypothetical protein TT1380 from Thermus thermophilus HB8. Proteins, 55, 778-780.

31. Chao, A., \& Goulding, C. W. (2019). A single mutation in the Mycobacterium tuberculosis heme-degrading protein, MhuD, results in different products. Biochemistry, 58(6), 489-492.

32. Sciara, G., Kendrew, S. G., Miele, A. E., Marsh, N. G., Federici, L., Malatesta, F., Schimperna, G., Savino, C., \& Vallone, B. (2003). The structure of ActVA-Orf6, a novel type of monooxygenase involved in actinorhodin biosynthesis. The EMBO Journal, 22(2), 205-215.

33. Larkin, M. A., Blackshields, G., Brown, N. P., Chenna, R., McGettigan, P. A., McWilliam, H., Valentin, F., Wallace, I. M., Wilm, A., Lopez, R., Thompson, J. D., Gibson, T. J., \& Higgins, D. G. (2007). Clustal W and Clustal X version 2.0. Bioinformatics, 23(21), 2947-2948.

34. Robert, X., \& Gouet, P. (2014). Deciphering key features in protein structures with the new ENDscript server. Nucleic Acids Research, 42(Web Server issue), 320-324.

35. Li, L., Liu, X., Yang, W., Xu, F., Wang, W., Feng, L., Bartlam, M., Wang, L., \& Rao, Z. (2007). Crystal structure of long-chain alkane monooxygenase (LadA) in complex with coenzyme FMN: Unveiling the long-chain alkane hydroxylase. Journal of Molecular Biology, 376, 453-465.

36. Shima, S., Warkentin, E., Grabarse, W., Sordel, M., Wicke, M., Thauer, R. K., \& Ermler, U. (2000). Structure of coenzyme F420 dependent methylenetetrahydromethanopterin reductase from two methanogenic archaea. Journal of Molecular Biology, 300, 935-950.

37. Oyugi, M. A., Bashiri, G., Baker, E. N., \& Johnson-Winters, K. (2016). Investigating the reaction mechanism of F420-dependent glucose-6-phosphate dehydrogenase from Mycobacterium tuberculosis: Kinetic analysis of the wild-type and mutant enzymes. Biochemistry, 55, 5566-5577.

38. Bashiri, G., Squire, C. J., Moreland, N. J., \& Baker, E. N. (2008). Crystal structures of F420-dependent glucose-6-phosphate 
dehydrogenase FGD1 involved in the activation of the antituberculosis drug candidate PA-824 reveal the basis of coenzyme and substrate binding. Journal of Biological Chemistry, 283(25), 17531-17541.

39. Greening, C., Ahmed, F. H., Mohamed, A. E., Lee, B. M., Pandey, G., Warden, A. C., Scott, C., Oakeshott, J. G., Taylor, M. C., \& Jackson, C. J. (2016). Physiology, biochemistry, and applications of F420- and Fo-dependent redox reactions. Microbiology and Molecular Biology Reviews, 80(2), 451-493.

40. Eichhorn, E., Davey, C. A., Sargent, D. F., Leisinger, T., \& Richmond, T. J. (2002). Crystal structure of Escherichia coli alkanesulfonate monooxygenase SsuD. Journal of Molecular Biology, 324, 457-468.

41. Campbell, Z. T., Weichsel, A., Montfort, W. R., \& Baldwin, T. O. (2009). Crystal structure of the bacterial luciferase/flavin complex provides insight into the function of the $\beta$ Subunit. Biochemistry, 48(26), 6085-6094.

42. Maier, S., Pflüger, T., Loesgen, S., Asmus, K., Brötz, E., Paululat, T., Zeeck, A., Andrade, S., \& Bechthold, A. (2014). Insights into the bioactivity of mensacarcin and epoxide formation by $\mathrm{MsnO8}$. ChemBioChem, 15, 749-756.

43. Sottorff, I., Künzel, S., Wiese, J., Lipfert, M., Preußke, N., Sönnichsen, F. D., \& Imhoff, J. F. (2019). Antitumor anthraquinones from an Easter Island Sea Anemone: animal or bacterial origin? Marine Drugs, 17, 154.

44. Okamoto, S., Taguchi, T., Ochi, K., \& Ichinose, K. (2009). Biosynthesis of actinorhodin and related antibiotics: Discovery of alternative routes for quinone formation encoded in the act gene cluster. Chemistry \& Biology, 16, 226-236.

Publisher's Note Springer Nature remains neutral with regard to jurisdictional claims in published maps and institutional affiliations. 